\title{
Responsabilidad social empresarial y su impacto en la disposición a pagar más entre los consumidores colombianos
}

\author{
López Celis, Diana María ${ }^{1}$ \\ Peñalosa Otero, Mónica Eugenia²
}

\section{Resumen}

El propósito de este trabajo es determinar la influencia que tienen o podrían llegar a tener los programas de Responsabilidad Social Empresarial,en la disposición de los consumidores a pagar más por las marcas y/o productosque realicen prácticas de responsabilidad social empresarial, evaluando dentro de la responsabilidad social empresarial, las dimensiones de cuidado ambiental, cuidado social/ético y trato con los empleados. El tipo de investigación fue concluyente con alcance cuantitativo, la mediciónse hizo con base en la batería de frases actitudinales correspondientes a la responsabilidad social empresarial tomados de Socially Resposible Purchase and Disposal (Webb et al., 2008); el análisis se realizó a través de regresiones logísticas, donde se logró determinar un modelo de influencia para las tres dimensiones de responsabilidad social empresarial evaluadasy su impacto en la disposición de los consumidores a pagar más por estas marcas/productos. El estudio arrojó un aporte positivo de las 3 dimensiones de la responsabilidad social empresarialen la disposición de los consumidores colombianos a pagar más; con mayor aporte de la variable ambiental, respecto a las variables social y ética, lo cual contribuye a sustentar el uso de las dimensiones de laresponsabilidad social empresarial en los planes de mercadeo, además;sustenta el impacto positivo que puede tenerpara las empresas,no solamente en las ventas (disposición a pagar más),también en la construcción de marca.

Palabras Clave: Responsabilidad social empresarial; regresión logística; disposición a pagar más.

\footnotetext{
Recibido: 15.01.19 Aceptado: 20.03.19
}

1 Magíster en Psicología, especialista en Psicología del Consumidor y sicóloga de la Universidad Konrad Lorenz, Bogotá. Docente Asociado II. Investigadora Junior (IJ) avalada por Colciencias. Universidad Jorge Tadeo Lozano - Bogotá, Colombia. diana.lopez@utadeo.edu.co

2 Magister en Mercadeo Agroindustrial y Profesional en Relaciones Internacionales de la Universidad Jorge Tadeo Lozano. Docente Asociado II. Investigadora Junior (IJ) avalada por Colciencias. Universidad Jorge Tadeo Lozano - Bogotá, Colombia. monica.penalosa@utadeo.edu.co 


\title{
Corporate social responsibility and its impact on the willingness to pay more among Colombian consumers
}

\begin{abstract}
Abstrac
The purpose of this paper is to determine the influence that the Corporate Social Responsibility (CSR) programs have or could have on the willingness of consumers to pay more for brands and / or products that carry out corporate social responsibility practices. evaluating within the RSE the dimensions of environmental care, social / ethical care and dealing with employees. The type of research was conclusive with quantitative scope, the measurement was made based on the battery of attitudinal phrases corresponding to the RSE taken from Socially Resposible Purchase and Disposal (SRPD) (Webb et al., 2008); the analysis was carried out through logistic regressions, where it was possible to determine a model of influence for the three dimensions of (CSR) evaluated and their impact on the willingness of consumers to pay more for these brands / products. The study showed a positive contribution of the 3 dimensions of the (CSR) in the willingness of Colombian consumers to pay more; with greater contribution of the environmental variable, with respect to the social and ethical variables, which contributes to sustain the use of the dimensions of the (CSR) in the marketing plans, in addition; it sustains the positive impact it can have for companies, not only on sales (willingness to pay more), but also on brand building.
\end{abstract}

Keywords: Corporate social responsibility; logistic regression; willingness to pay more.

\section{Introducción}

El mercadeo dentro de su dinámica cambiante, evolutiva y adaptativa a las diferentes formas de pensar, sentir y actuar del consumidor frente a las marcas, ha venido presentando una nueva tendencia, donde este consumidor y su dinámica con las marcas ha modificado conductas.

Actualmente el mercadeo no es un monólogo sino una conversación totalmente participativa y de doble vía; donde el consumidor va más allá de un simple vínculo con la marca porque es la líder del mercado o porque genera estatus, el vínculo que genera la marca con el consumidor, se encuentra dado por valores agregados que reposan más a nivel funcional, el consumidor ha dejado de pensar individualmente y ha empezado a pensar más de forma colectiva; es decir lo que más conviene para él mismo y el mundo, entendido como el todo.

Hoy, más que en ningún otro momento es importante para el consumidor saber a favor de qué o en contra de qué convive una marca; para los consumidores es importante conocer 
y entender en qué nivel las marcas están o no comprometidas con el todo, con la comunidad, hasta dónde su razón de ser va más allá de satisfacer una necesidad a través de un producto y lograr un posicionamiento.

Existen estudios previos donde ya se mencionan las principales variables que el consumidor tiene en cuenta al adquirir un producto; tales como el precio, la cercanía con el cliente, el servicio, entre otras; pero a medida que el consumidor deja de pensar en forma individual y empieza a pensar de forma colectiva, surgen otras variables a considerar tal como es el caso de la dimensión de consumo responsable, la cual influye en el proceso de decisión y elección de las marcas.

De acuerdo con Havas Worldwide (2016), se encuentra que el $58 \%$ de los consumidores se interesan por el impacto social y ambiental de las marcas. Lo anterior, indica que los nuevos consumidores y las nuevas generaciones, lideran una nueva tendencia en los modelos de negocio, por lo tanto, existe una nueva generación de consumidores mucho más exigentes, participativos y con una conciencia colectiva, respecto a las actividades medioambientales y de responsabilidad social de las compañías, (Mohr \& Webb, 2005; Lecompte \& Roberts, 2006; Cai \& Aguilar, 2013; Cone Study, 2013).

El consumidor ahora más que nunca valora el respeto, la transparencia y la conciencia de las marcas, los consumidores buscan marcas en las cuales puedan creer y les generen confianza, les interesa conocer cómo y con qué insumos son fabricadas, cómo estos productos pueden afectar o beneficiar la salud, cuáles son sus prácticas laborales, cuál es su compromiso con el medio ambiente y cómo las ganancias de las empresas apoyan al consumidor o le retribuyen su aceptación o participación en las marcas; es decir, poder conocer con claridad el manejo que le dan a sus ganancias.

De otro lado y de acuerdo con el Foro Económico Mundial, realizó la encuesta Global Shapers Survey en el 2017 en 180 países, con el fin de conocer las percepciones de los jóvenes acerca del mundo, se pudo ratificar que los llamados millennials o jóvenes que tienen actualmente entre 18 y 35 años, están preocupados, especialmente, por los efectos que ha tenido el cambio climático en el medio ambiente. Es así, como de los 31.000 encuestados un $48,8 \%$ respondió que el cambio climático y la destrucción de la naturaleza era uno de los asuntos más graves a nivel global. A este problema le siguen los grandes conflictos sociales con un $38,9 \%$, y en tercer lugar se ubica la desigualdad y la discriminación con un $30,8 \%$. En total fueron 31.000 jóvenes los encuestados según el World Econmic Forum (2017).

Lo anterior supone una generación con mayor conciencia ambiental, lo que significa que están cambiando sus hábitos de compra y están siendo analíticos frente a sus acciones con el medio ambiente. Es por esto, que han comenzado a ahorrar agua y energía, reciclar y buscar medios de transporte ecoeficientes como planes preventivos para mitigar los impactos negativos.

Lo cierto es, que el conocimiento que tienen los consumidores sobre el significado de la responsabilidad social y el impacto que esta pueda generar en las decisiones de compra, es un tema relativamente nuevo que comienza a cobrar importancia en el contexto actual $y$, por consiguiente, las empresas se enfrentan a un mayor reto frente a la problemática de la responsabilidad 
social y ambiental en sus actividades; dado que son conceptos que muchas empresas vienen apenas entendiendo y adoptando (Webb et al, 2008).

De acuerdo a lo anterior, con este trabajo se busca dar respuesta a la siguiente pregunta: ¿Influyen los programas de responsabilidad social empresarial en la disposición de los consumidores a pagar más por marcas/ productos que realicen prácticas de responsabilidad social empresarial? Para tal fin, se estableció como objetivo determinar la influencia de los programas de responsabilidad social empresarial en Colombia, en la disposición de los consumidores a pagar más por estas marcas $y / o$ productos que realicen prácticas de responsabilidad social empresarial.

El enfoque de la presente investigación es causal con un alcance cuantitativo, basado en la aplicación de encuestas a nivel nacional para la recogida de la información, el trabajo de campo fue realizado por la empresa B\&Optimos durante el segundo semestre de 2015, con un muestreo no probabilístico en centros de gran afluencia.

Las variables de RSE objeto de estudio en la presente investigación, se midieron a través de escalas (SRPD), de la cual a juicio de expertos se seleccionaron las frases actitudinales de mayor relación con el tema, adaptándolas al contexto y cultura colombiana, mediante los correspondientes pretest cualitativos y cuantitativos; se tomaron dos frases actitudinales de la escala para medir la dimensión ambiental, tres para la dimensión ética_prácticas laborales y una para la dimensión ambiental, frases actitudinales que fueron tratadas cada una como variables independientes, para un total de 6 variables. Dichas variables fueron incluidas en un cuestionario que se aplicó a una muestra de 400 participantes de las principales ciudades del país (Bogotá, Medellín, Cali y Barranquilla), mayores de 18 años del nivel socioeconómico del 3 al 6 , implementando un diseño muestral estratificado por género y nivel socioeconómico.

El estudio se realizó con una confiabilidad del $95 \%$ y $5 \%$ de margen de error, teniendo en cuenta el Universo total de la población colombiana según el DANE fue de 48.010 .049 personas, distribuidas proporcionalmente para cada ciudad; 100 en Cali, 100 en Bogotá, 100 en Barranquilla y 100 en Medellín. Esta muestra garantizó la representatividad de la muestra en los términos aceptados y validados de acuerdo con la literatura (Román \& Cuestas, 2008; Román, 2010). Para la medición de los constructos, se empleó una escala multi-ítem tipo Likert siguiendo un formato de 5 puntos Likert determinados.

\section{Contextualización de la Responsabilidad Social Empresarial.}

La responsabilidad social es una variable que tiene gran relevancia para el consumidor Miles y Munilla (2004), indican que esta variable en su dinámica puede formar parte importante del plan de mercadeo, los autores referencian, por ejemplo, que los consumidores estarían dispuestos a pagar un sobreprecio por los productos de empresas que tienen un trato socialmente responsable hacia sus trabajadores.

Es importante considerar que en el análisis de la responsabilidad social empresarial RSE, los atributos sociales ejercen un papel importante 
en el proceso de decisión y elección de compra del consumidor, siendo la variable que puede llevar a decidir entre una u otra marca, en algunos casos cobrando más relevancia frente a otros atributos intangibles como pueden ser el país de origen o la marca (Auger et al, 2010:157).

Para el caso colombiano, al realizar la revisión teórica, solamente se encontraron 4 investigaciones respecto a la dinámica que tiene la RSE en la decisión de compra; uno realizado en la ciudad de Bogotá "Impacto de la responsabilidad social empresarial en el comportamiento de compra y disposición a pagar de consumidores bogotanos" (Percie y Reficco 2015), "Disposición a pagar por atributos de responsabilidad social empresarial Un estudio aplicado a jóvenes de Medellín" (Solari, E. \& Pacheco Ortiz, D 2015) y "Diseño y Responsabilidad Social Empresarial en la planeación estratégica de la marca", realizado como artículo de reflexión por autores Colombianos (Alzate y Orozco 2015); estos 3 estudios son coincidentes en sus resultados, concluyen que los consumidores consideran el atributo de responsabilidad social en su decisión de compra y que el manejo de la RSE dentro de la planeación estratégica ayuda en la construcción de marca, cabe anotar que en la revisión teórica realizada no se encontraron estudios a nivel nacional en Colombia, como se plasma en el documento se encontró para ciudades específicas, cobrando relevancia el hecho de que esta investigación se haya realizado teniendo en cuenta una cobertura nacional, contemplando las principales ciudades de acuerdo a su distribución poblacional (Bogotá, Cali, Medellín y Barraquilla).

En Colombia los programas de responsabilidad social empresarial se implementan por convicción de sus directivas o normas legales, pero se hace necesario conocer no solamente por ciudades geográficas o grupos específicos sino a nivel país, la predisposición de los consumidores a pagar más por productos que implementen prácticas de RSE, y que esta variable se convierta en una estrategia atractiva para las empresas colombianas como aporte de construcción a sus marcas, más allá de ser considerada simplemente una norma más; también puede aumentar el número de empresas comprometidas con los programas de RSE y las que ya implementen estos programas, puedan obtener un mayor beneficio, a través de la integración de este atributo en la planeación estratégica de mercadeo, puesto que actualmente en las nuevas generaciones de consumidores, los temas sociales, ambientales y éticos tienen gran relevancia (Cone Study, 2013; Tully \& Winer, 2014), al mismo tiempo trabajar en la construcción de una comunicación impactante y atractiva para los consumidores que han integrado a sus repertorio conductual la variable del consumo responsable, para esta generación emergente con características distintivas (Cone Study, 2013), siendo importante para la competitividad de las empresas colombianas.

El consumo socialmente responsable (CSR) y la responsabilidad social empresarial RSE constituyen dos caras de una misma moneda denominada consumo sostenible (Lorek \& Spangenberg, 2014). Para Wymer y Rundle-thiele (2017), la RSE es una noción mediante la cual las organizaciones reflejan los intereses de la sociedad al asumir la responsabilidad del impacto de sus actividades en 
una amplia variedad de stakeholders, entre quienes incluyen a los clientes, proveedores, empleados, accionistas, comunidades y el medioambiente. El CSR rechaza los productos riesgosos para la salud, los productos con empaques no ecológicos o que desperdician energía, los productos que utilizan materiales peligrosos o provienen de hábitats o especies en peligro. Así mismo, el CSR evalúa a las empresas en su comportamiento responsable y en el comercio justo.

Actualmente el consumidor, es considerado como más funcional, dado que no solamente tiene en cuenta la satisfacción básica a través de la compra de una producto, sino que considera aspectos medioambientales, éticos e involucra otras variables específicas como la responsabilidad social de las empresas y el contexto socioeconómico y cultural, donde la información trasciende más allá de los propios bienes y servicios (Mohr et al., 2001; Ozcaglar-Toulouse, 2005; Webb et al., 2008), citados por (Dueñas \& Perdomo 2014). Ahora más que nunca es importante revisar al consumidor desde otra óptica, considerando no solamente las variables sociales, medioambientales y de responsabilidad social de las empresas, ahora es fundamental comprender el contexto económico y social del consumidor (Dueñas et al., 2014). La información que el consumidor procesa, con la cual busca una interacción de doble vía con las marcas, donde el adquirir productos y servicios socialmente responsables, direcciona en gran medida su consumo, el consumo responsable empieza a ser tenido en cuenta como un fenómeno colectivo y dependiente de los grupos sociales con los que el consumidor interactúa (Dueñas et al, 2014). Por consiguiente, Hailes (2007) y Akehurst et al, (2012) definen al CSR en el contexto del nuevo siglo como aquel que ve en sus actos de consumo la opción y oportunidad de proteger y preservar el medio ambiente y la calidad de vida en conjunto, ya no desde el punto de vista individual sino colectivo, es así como el estudio llevado a cabo por (Cone Study, 2013) sobre Consumo Socialmente Responsable (CSR) reporta que "casi el $90 \%$ de los americanos, indican que actualmente es más importante que nunca para las empresas ser socialmente responsables".

\subsection{Dimensiones y medición de la Responsabilidad Social Empresarial RSE}

La Responsabilidad Social Empresarial reconoce diferentes ámbitos que responden a los compromisos que, por ley, debe cumplir una empresa para que sea reconocida como tal, así como a sus acciones voluntarias en el mejoramiento de las condiciones sociales, vistas desde diferentes contextos: el ambiental, el económico y el social. Para Carroll (1979), Alvarado \& Schlesinger (2008) y Cancino \& Morales (2008) existen cuatro dimensiones de la Responsabilidad Social Empresarial económica, legal, ética y discrecional o filantrópica, las cuales reúnen las diferentes obligaciones que deben cumplir las empresas que puedan considerarse socialmente responsables.

La dimensión económica se refiere a la labor específica de la empresa, que la hace rentable; la dimensión legal aborda las obligaciones jurídicas; la dimensión ética establece las normas que se deben cumplir con relación a las expectativas sociales y, la dimensión discrecional o 
filantrópica, se relaciona con las acciones voluntarias que se hacen para participar en la construcción de un entorno social más amable. Sin embargo, la dimensión discrecional o filantrópica de alguna forma genera mayor valor y conexión con los consumidores, pues además de ser voluntaria puede crear mayor visibilidad en la comunicación de las acciones. Para Alvarado \& Schlesinger (2008), esta dimensión influye directamente en la imagen y la reputación de la empresa, mientras que las dimensiones ético-legal son de segundo orden y la económica no es tan relevante desde el punto de vista del consumidor.

La última década ha sido altamente productiva en la medición del Consumo Socialmente Responsable (CSR). De acuerdo con Mohr et al. (2005), se identifica un importante vacío en la medición, el cual estaba ya anticipado por Kinnear \& Taylor (1973), representado en la ausencia de una dimensión que diera cuenta del efecto de la RSE sobre el (CSR). De la misma manera, se reconoce la importancia de la segmentación del mercado según el tipo de consumidores responsables, llegando al desarrollo de una nueva escala, la Socially Resposible Purchase and Disposal (SRPD) (Webb et al, 2008). La escala SRPD se conforma de 4 dimensiones: "una dimensión del efecto de la RSE sobre el consumo denominada "desempeño en responsabilidad social corporativa"; una dimensión sobre el reciclaje y manejo de desperdicios; una dimensión relacionada con la reducción del uso de determinados productos o servicios que tienen impacto sobre el medio ambiente, y una dimensión de ítems liberales que reincorpora la visión de la personalidad responsable en las decisiones de compra.

\subsection{La asociación entre la responsabilidad social empresarial y la disposición de los consumidores a pagar más.}

El impacto de la RSE en las marcas, no solamente ha sido objeto de estudio para la cultura occidental, también es tema de investigación para la cultura oriental, En el ámbito del mercadeo, Tian, Wang \& Yang (2011), encontraron que "los consumidores con un mayor nivel de conocimiento y confianza de la RSE de las empresas, eran más propensos a pasar de una evaluación positiva de ellas a una intención de compra positiva"; por otra parte, diferentes estudios han encontrado que al parecer es mucho más frecuente encontrar que las mujeres apoyen las causas de RSE, tal como lo han documentado Arredondo, Maldonado \& de la Garza (2011), a la hora de analizar sus percepciones y actitudes de compra en el marco de campañas de marketing con causa.

Estos hallazgos soportan el impacto positivo del uso de los programas de RSE, citados por (Marquina Feldman \& Reficco, 2015:375), así como la investigación llevada a cabo por Cai \& Aguilar (2013), en donde se identificó una predisposición positiva a pagar precios extra por productos social y ambientalmente certificados, específicamente, estarían dispuestos a pagar un $12,2 \%$ más de su valor real por este tipo de productos. A nivel global los estudios señalan que los consumidores trasladan su percepción sobre la responsabilidad de las empresas a sus decisiones de compra. Marquina Feldman \& Reficco, (2015), demuestran que el $65 \%$ de los encuestados sienten responsabilidad para adquirir productos 
positivos para el medio ambiente y la sociedad.

En el 2015 y de acuerdo con la última medición llevada a cabo entre las principales empresas colombianas, se refleja que cuando se pregunta sobre el valor que aporta la RSE a la empresa, respecto a la reputación/valor de marca, el $22,3 \%$, considera que aporta a la construcción de marca, según la Asociación Nacional de Empresarios de Colombia. Igualmente, de acuerdo con la revisión realizada por el sector privado colombiano, se puede apreciar cómo Colombia ha adoptado los programas de RSE más como una variable de cumplimiento legal cercana a la gestión de riesgos y/o reputacional, en lugar de considerar esta variable como un atributo de diferenciación frente al consumidor.

Para Castelló (2010), la RSE debe ser parte integral de las estrategias de branding corporativo, pues la buena relación con el sector social puede repercutir finalmente en el aspecto económico. Según Villafañe (2009), "la RSE se ha convertido en parte importante de la comunicación y construcción de marca, puesto que, en la medida en que se gestionen adecuadamente los diferentes aspectos que hacen parte de la relación empresa-comunidad o marca-comunidad, es posible generar una imagen positiva en la mente de los consumidores", citados por (Alzate, J. E., \& Orozco-Toro, J. A. (2015:141).

Este estudio no persigue desvirtuar o poner en entredicho las variables que típicamente la literatura y las diferentes investigaciones de marketing, han demostrado que influyen en la conducta de compra del consumidor, se busca dar un aporte respecto a las nuevas variables que debido al cambio generacional y cultural impactan al consumidor, y su incidencia en el proceso de decisión y elección de las marcas.

Finalmente, para Licandro, Alvarado, Sansores \& Navarrete (2019), la interpretación que tienen los tomadores de decisiones en las empresas sobre el concepto de RSE, es un factor crítico para su incorporación a la praxis empresarial; de esa interpretación depende el grado de transversalidad con el que se lo aplica y la forma como se lo gestiona.

Por lo tanto, se encuentra apoyo teórico para sustentar que el consumo socialmente responsable (CSR) puede resultar rentable para las empresas, porque es una variable que el consumidor tiene en cuenta en el momento de realizar la compra; además, la literatura también reporta, que los consumidores están dispuestos a pagar más por productos que sean socialmente responsables.

\section{Incidencia de la dimensión; social, ética y ambiental en la disposición de los consumidores colombianos a pagar más.}

Teniendo en cuenta la Socially Resposible Purchase and Disposal (SRPD) (Webb et al, 2008), se tomó como referencia las dimensiones de la RSE; en línea con Carroll (1979, 1999); Alvarado \& Schlesinger (2008) y Cancino \& Morales (2008), quienes contemplan la existencia de cuatro dimensiones de la RSE: la económica, la legal, la ética y la discrecional o filantrópica. A su vez, este estudio evaluó 3 dimensiones en concordancia con la RSE.

El compromiso social, el ambiental y las prácticas laborales; correspondientes también a tres de las siete dimensiones que evalúa la 
ISO 26000 (participación y desarrollo de la comunidad, derechos humanos, prácticas laborales, medio ambiente, prácticas de justa operación, asunto de consumidores y gobernanza). Lo anterior, teniendo en cuenta que, en la revisión teórica, estas variables mostraron ser las de mejor desempeño e impacto en la disposición de los consumidores a pagar más por un producto o marcas, que manejen programas de responsabilidad empresarial.

En este sentido, se plantea como hipótesis de trabajo:

$\mathrm{H} 1$ : Existe influencia entre los programas de responsabilidad social empresarial y la disposición de los consumidores a pagar más por marcas I productos que realicen prácticas de responsabilidad social empresarial.

Como variables independientes, quedarían:

- Dimensión de compromiso ambiental

- Dimensión de compromiso social

- Dimensión de compromiso con los empleados_ético

Siendo la variable dependiente:

- Disposición a pagar más por productos por marcas que manejan programas de RSE.

Con respecto a la contrastación de Hipótesis H1, se procede a realizar un Ajuste del Modelo de Regresión Logística:

VD: Disposición a pagar más por productos responsables VS (dimensión ambiental (VI1, VI2); (dimensión ética trato con los empleados (VI3, VI4, VI5); dimensión social (VI6)

Una vez ajustado el modelo (tabla 1), se evidencia que se encuadra dentro de los denominados Modelos Lineales Generalizados (Hosmer \& Lemeshow, 2000), donde la variable dependiente (VD) se transformó en una variable dicotómica, donde los puntajes 4 y 5 señalan los éxitos (1), mientras que cualquiera de los demás posibles valores señalan fracaso $(0)$. El resultado del ajuste se presenta a continuación. (Tabla 1)

Tabla 1

Modelo de Regresión Logística

\begin{tabular}{lccc}
\hline Fuente & B & Chi-cuadrado de Wald & Pr $>\mathrm{Chi}^{2}$ \\
\hline Intercepción & $-7,8036$ & 76,5197 & $<0,0001$ \\
\hline VD1 & 0,5196 & 10,2308 & 0,0014 \\
\hline VD2 & 0,6863 & 16,1197 & $<0,0001$ \\
\hline VD3 & 0,1669 & 0,9745 & 0,3236 \\
\hline VD4 & 0,0287 & 0,0308 & 0,8608 \\
\hline VD5 & 0,1246 & 0,9435 & 0,03314 \\
\hline VD6 & 0,6447 & 18,4259 & $<0,0001$ \\
\hline
\end{tabular}

Fuente: Elaboración propia 
De la tabla anterior se puede deducir, según la Probabilidad asociada a las Pruebas del Chi2 (tabla 2), que las variables que más aportan en la explicación de (VD), son VD1 y VD2, correspondientes a compromiso ambiental y VD6 evaluando compromiso social, y en menor grado VD3, VD4 y VD5 concernientes a prácticas éticaslaborales. La constante del modelo tiene también un papel significativo en el ajuste de este modelo

\section{Tabla 2}

Prueba de la hipótesis nula H0: Y=0,5300 (VD_VI's)

\begin{tabular}{llll}
\hline \multicolumn{1}{c}{ Estadística } & GDL & \multicolumn{1}{c}{ Chi-cuadrado } & $\mathrm{Pr}>\mathrm{Chi}^{2}$ \\
\hline -2 Log(Verosimilitud) & 6 & 141,4458 & $<0,0001$ \\
\hline Score & 6 & 117,5440 & $<0,0001$ \\
\hline Wald & 6 & 84,9453 & $<0,0001$ \\
\hline
\end{tabular}

Fuente: Elaboración propia

El valor más importante es el estadístico Chi^2 asociado al Log Ratio (L.R.), esto es el equivalente de la prueba $\mathrm{F}$ de Fisher del modelo lineal; en este caso se desea evaluar si las variables independientes VD1, VD2, VD3, VD4, VD5 y VD6 proporcionan una cantidad de información significativa para explicar la variable binaria VD. En este caso, como la probabilidad es inferior a 0.0001 , se puede deducir que las variables proporcionan una cantidad significativa de información.

La última etapa reside en la aplicación del modelo ajustado. En este caso el modelo se escribe:

$$
Y=\frac{\operatorname{Exp}(L(x))}{1+\operatorname{Exp}(L(x))}
$$

(Ecuación 1)

Dónde $\quad L(x)=-7,8036+0,5196 * V I 1+0,6863 * V I 2+0,1669 * V I 3+$ $0,0287 * V I 4+0,1246 * V I 5+0,6447 * V I 6$

Desde el modelo teórico planteado, la Curva Característica de Poder (ROC) señala la eficiencia teórica del modelo para discriminar nuevos casos, la cual fue del $82.58 \%$. Diagrama 1 


\section{Diagrama 1 Eficiencia teórica del modelo}

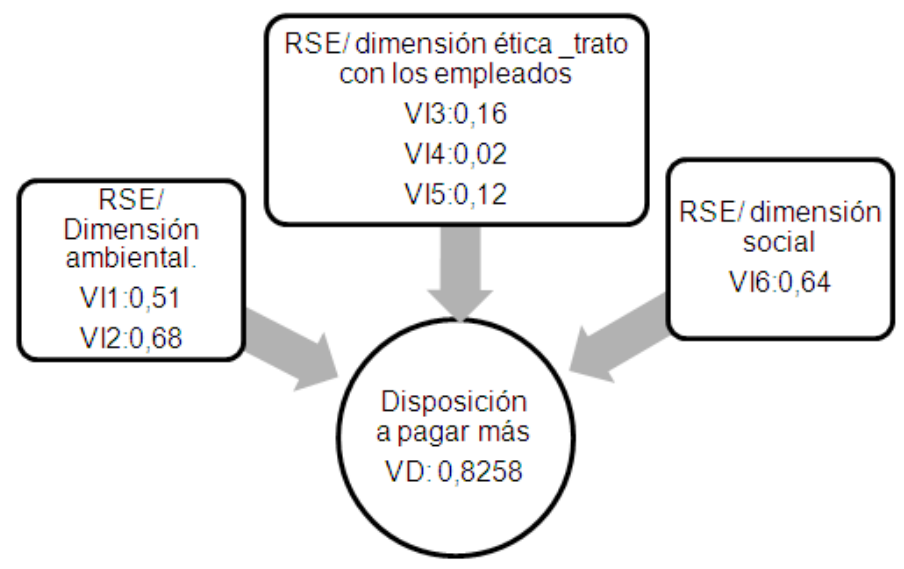

Fuente: Elaboración propia

\section{Conclusiones}

De la revisión teórica de este trabajo cabe destacar, la importancia que se le da al manejo de las dimensiones de RSE dado por el cambio generacional y llegar a ser considerado dentro del campo de las recientes tendencias, como un aporte a las nuevas variables que los consumidores han venido involucrando en su proceso de decisión y elección para las compras y su disposición a pagar más. Por lo anterior, es necesario sustentar a través de la investigación, si estas recientes tendencias van más allá de una moda y realmente están entrando a formar parte del repertorio conductual de los consumidores, aportando en una predisposición a pagar más por productos elaborados por empresas que realicen prácticas de RSE y por consiguiente, contribuyendo a la creación de un vínculo con la marca, lo cual hace atractivo e importante involucrar estrategias de RSE en los planes de mercadeo de las empresas, de acuerdo al hallazgo de esta investigación.

Ahora bien, no debe considerarse el incorporar la variable de RSE como un todo en los planes de mercadeo, teniendo en cuenta que ésta contempla diferentes dimensiones y que pueden impactar de diferentes maneras al consumidor en su disposición a pagar más. De acuerdo a lo anterior, en este estudio se evidencia que en general las dimensiones social, ética y ambiental en su conjunto, aportan a esta conducta, sin embargo, contribuye de manera principal la dimensión ambiental, seguida de la social y en tercer lugar la dimensión ética; lo cual explica que no puede hacerse referencia global a la integración de la RSE en los planes de mercadeo y se hace necesario especificar, cuál o cuáles son las variables a manejar por 
parte del sector empresarial.

Es importante continuar en la profundización del tema a través de otras técnicas de investigación, como las sesiones de grupo o las entrevistas en profundidad, que conduzcan a una comprensión, acerca de las razones por las cuales la dimensión ambiental y social son las que más aportan a pagar más, mientras que la variable ética no es de mayor consideración; esto debido posiblemente a que los consumidores no la conocen, o no es implementada por las empresas, o no tiene difusión, como si pueden presentarse con las otras dimensiones.

Finalmente, se hace necesario trabajar en una estrategia de comunicación para las dimensiones que generan mayor disposición a pagar más; es decir, la social y la ambiental; puesto que el estudio arroja certeza sobre la influencia que tienen; por lo tanto, hay que investigar acerca de la mejor forma de ser adherida en los planes de mercadeo empresariales.

\section{Referencias Bibliográficas}

Akehurst, Gary; Afonso, Carolina \& Martins, Helena. (2012), Re-examining green purchase behavior and the green consumer profile: new evidences. Management Decisión, 50 (5), pp. 972-988. Disponible en: https://www.emeraldinsight.com/doi/ full/10.1108/00251741211227726

Alvarado, Herrera; Alejandro, Schlesinger; Díaz, María Walesska. (2008), Dimensionalidad de la Responsabilidad Social Empresarial percibida y sus efectos sobre la imagen y la reputación: Una aproximación desde el modelo de Carroll. Estudios Gerenciales, [en linea] 24 (Julio-Septiembre) : [Fecha de consulta: 20 de junio de 2019] Disponible en:<http://www.redalyc.org/articulo. oa? id=21210802> ISSN 0123-5923

Alzate, Jaime \& Orozco, Jaime Alberto. (2015), Diseño y Responsabilidad Social Empresarial en la planeación estratégica de la marca. Revista Kepes, 12, pp. 133-155.

Arredondo, Florina Guadalupe; Maldonado, Verónica del Carmen \& De La Garza, Jorge. (2010). Consumers and their buying decision making based on price and information about corporate social responsibility (CSR). Case study: Undergraduate students from a private university in Mexico. Estudios Gerenciales, 26 (117), pp. 103-117. Disponible en: http:// www.scielo.org.co/pdf/eg/v26n117/ v26n117a06.pdf

Auger, Pat \& Devinney, Timothy \& Louviere, Jordan \& Burke, Paul. (2009). The importance of social product attributes in consumer purchasing decisions: A multi-country comparative study. International Business Review, 19. pp. 140-159. 10.2139/ ssrn.1270474.

Cai, Zhen and Francisco X. Aguilar (2013), "Meta-analysis of Consumer's Willingness-to-Pay Premiumsfor Certified Wood Products," Journal of Forest Economics, 19 (January), pp. 15-31.

Cancino, Christian \& Morales, Mario. (2008), Responsabilidad social empresarial. Serie Documento Docente. Universidad de Chile. Santiago de Chile, Chile. Disponible en: http://repositorio.uchile.cl/bitstream/ handle/2250/122747/Cancino_Morales_2008.pdf?sequence $=1$

Carroll, Archie. (1979), A Three-Dimensional Conceptual Model of Social Performance. Academy of Management Review. 4. 497-505. $10.2307 / 257850$. 
Responsabilidad social empresarial y su impacto en la disposición a pagar más entre los consumidores colombianos

López Celis, Diana María y Peñalosa Otero, Mónica Eugenia

Carroll, Archie. (2015), Corporate social responsibility: The centerpiece of competing and complementary frameworks. Organizational Dynamics, 44, pp. 87-96

Cone Study. (2013). Cone Communications Social Impact Study: The nex cause evolution. Disponible en: Cone Communications Study: http://www.conecomm.com/stuff/ contentmgr/files/0/e3d2eec1e15e858867a5c2b1a22c4cfb/files/2013 cone_comm_social_impact_study. pdf

Dueñas, Sebastián; Perdomo-Ortiz, Jesús \& Villa-Castaño, Lida. (2014), El concepto de consumo socialmente responsable y su medición. Una revisión de la literatura. Estudios Gerenciales [en linea] 30 (Julio-Septiembre) Disponible en:<http://www.redalyc.org/articulo. oa? id=21231380009> ISSN 01235923 Fecha de consulta: 20 de junio de 2019

Hailes, Julia. (2007), The New Green Consumer Guide. UK: Simon y Schuster Ltd.

Havas Worldwide (2016). Superbrands 2016: los consumidores $y$ el futuro de los modelos empresariales. Disponible en: http:// www.compromisoempresarial.com/ rsc/2016/05/el-58-de-los-consumidores-se-interesa-por-el-impacto-social-y-ambiental-de-las-marcas/

Kinnear, Thomas \& Taylor, James. (1973), The Effect of Ecological Concern on Brand Perceptions. Journal of Marketing Research, 10. 191. 10.2307/3149825.

Francois-Lecompte, Agnes \& Roberts, James A. (2006), Developing a measure of socially responsible consumption in France. Marketing Management Journal, 16 (2), pp. 50-66.
Hosmer David \& Lemeshow Stanley. (2000), Applied logistic regression. Segunda Edición. Wiley Series in Probability and Statistics.

Licandro, Oscar; Alvarado-Peña, Lisandro; Sansores-Guerrero, Edgar \& Navarrete, Juana. (2019), Responsabilidad Social Empresaria: Hacia la conformación de una tipología de definiciones. Revista Venezolana de Gerencia, 24. pp. 281-299. Recuperado en: http://produccioncientificaluz. org/index.php/rvg/article/view/23843 Fecha de consulta:20/06/2019

Lorek, Sylvia \& Spangenberg, Joachim. (2014), Sustainable consumption within a sustainable economy - Beyond green growth and green economies. Journal of Cleaner Production. 63. pp. 33-44. 10.1016/j. jclepro.2013.08.045.

Marquina, Feldman \& Percy-Reficco, Ezequiel. (2015), Impacto de la responsabilidad social empresarial en el comportamiento de compra y disposición a pagar de consumidores bogotanos. Estudios Gerenciales [en línea] 31 (octubre-diciembre): Disponible en:<http://www.redalyc.org/ articulo.oa?id=21243557003> ISSN 0123-5923 Fecha de consulta: 20 de junio de 2019

Miles, Morgan \& Munilla, Linda. (2004), "The potential impact of social accountability certification on marketing: A Short Note," Journal of Business Ethics, 50 (1): pp. 1-11.. Journal of Business Ethics. 50. 1-11. 10.1023/B:BUSI.0000021139.94166.74.

Mohr, Lois \& Webb, Deborah. (2005), The effects of corporate social responsibility and price on consumer responses. Journal of Consumer Affairs. 39. pp. 121 - 147. 10.1111/j.1745-6606.2005.00006.x.

Mohr, Lois; Webb, Deborah \& E. Harris, 
Katherine. (2005), Do Consumers Expect Companies to Be Socially Responsible? The Impact of Corporate Social Responsibility on Buying Behavior. Journal of Consumer Affairs. 35. pp. 45-72. 10.1111/j.17456606.2001.tb00102.x.

Ozcaglar-Toulouse Nil. (2005), Apport du concept d'identité à la compréhension ducomportement du consommateur responsable: Une application à la consom-mation des produits issus du commerce équitable. Université du Droit et dela Santé - Lille II Disponible en: http:// tel.archives-ouvertes.fr/tel-00300664

Solari, Estefanía. \& Pacheco Ortiz, Diana. (2015), Disposición a pagar por atributos de responsabilidad social empresarial. Un estudio aplicado a jóvenes de Medellín. Revista Virtual Universidad Católica del Norte, 46, pp. 187- 199. Disponible en: http:// revistavirtual.ucn.edu.co/index.php/ RevistaUCN/article/view/708/1235. ISSN 0124-5821Fecha de consulta: 20/06/2019

Tian, Zhilong; Wang, Rui \& Yang, Wen. (2011), Consumer responses to Corporate Social Responsibility. (CSR) in China. Journal of Business Ethics, 101, pp. 197-212. Disponible en: https://uu.diva-portal.org/smash/get/
diva2:1134668/FULLTEXT01.pdf

Tully, M, Stephanie \& Winer, Russell. (2014), The Role of the Beneficiary in Willingness to Pay for Socially Responsible Products: A Meta-analysis. Journal of Retailing, 90 (2), pp. 255-274.

Villafañe, Julio. (2009), Reputación corporativa y RSC. Bases empíricas para un análisis. Revista Telos, 79, pp. 75-82.

Webb, Deborah \& Mohr, Lois \& E. Harris, Katherine. (2008). A re-examination of socially responsible consumption and its measurement. Journal of Business Research. 61. pp. 91-98. 10.1016/j.jbusres.2007.05.007.

Wymer, Walter \& Rundle-thiele, Sahyn (2017), Inclusion of ethics, social responsibility, and sustainability in business school curricula: a benchmark study. International Review of Public and Nonprofit Marketing, 14, pp. 19-34. Recuperado en: https://doi. org/10.1007/s12208-016-0153-z Fecha de consulta:22-04-2018

World Econmic Forum. (2017), The Global Shapers Survey. \#shaperssurvey. Recuperado en: http://www. shaperssurvey2017.org/static/data/ WEF_GSC_Annual_Survey_2017. pdf Fēcha de consulta:20/06/2019

- $\quad$ Esta obra está bajo una licencia de Creative Commons Reconocimiento-NoComercial- Compartirlgual 3.0 Unported. http://creativecommons.org/licenses/by-nc-sa/3.0/deed.es_ES 